\title{
On the Maximum Principle for Impulsive Hybrid Systems
}

\author{
Vadim Azhmyakov ${ }^{1}$, Sid Ahmed Attia ${ }^{2}$, and Jörg Raisch ${ }^{2,3}$ \\ 1 Departamento de Control Automatico, CINVESTAV, A.P. 14-740, Av. Instituto \\ Politecnico Nacional No. 2508, C.P. 07360, Mexico D.F., Mexico \\ \{vazhmyakov@ctrl.cinvestav.mx\} \\ 2 Fachgebiet Regelungssysteme, Technische Universität Berlin, Einsteinufer 17, \\ D-10587 Berlin, Germany \{attia@ieee.org\} \\ 3 Systems and Control Theory Group, MPI for Dynamics of Complex Technical \\ Systems, Sandtorstr. 1, D-39106 Magdeburg, Germany \\ \{raisch@control.tu-berlin.de\}
}

\begin{abstract}
In this contribution, we consider a class of hybrid systems with continuous dynamics and jumps in the continuous state (impulsive hybrid systems). By using a newly elaborated version of the Pontryagintype Maximum Principle (MP) for optimal control processes governed by hybrid dynamics with autonomous location transitions, we extend the necessary optimality conditions to a class of Impulsive Hybrid Optimal Control Problems (IHOCPs). For these problems, we obtain a concise characterization of the Impulsive Hybrid MP (IHMP), namely, the corresponding boundary-value problem and some additional relations. As in the classical case, the proposed IHMP provides a basis for diverse computational algorithms for the treatment of IHOCPs.
\end{abstract}

Key Words: impulsive hybrid control systems, optimal control, necessary conditions of optimality, Maximum principle.

\section{Introduction}

During the last two decades, there has been considerable effort to develop theoretical and computational frameworks for hybrid systems. Of particular importance is the ability to operate such systems in an optimal manner. With the exception of certain special cases, the solution to the optimal problem remains a challenging task. This is due to the fact that the two aspects of system behaviour, i.e., discrete and continuous, are tightly linked, to such an extent that they cannot be decoupled in an effective and simple way. One of the most convenient ways to deal with the problem is to formulate it as a sequential problem, i.e., for a particular execution the time axis is partitioned into intervals, and in each interval, the dynamics are characterized by a set of ODEs, with transitions being triggered internally (autonomous switches) or externally (controlled 
switches). This is the approach that has been considered since the initial formulation of the corresponding optimal control problem $[15,16],[32]$ and can be seen as a natural way to tackle the problem. For a deeper discussion on the main theoretical questions see e.g., [2, 3, 6, 7, 12-14, 17, 18, 21, 27, 28, 32, 35].

The class of hybrid systems considered in this contribution involves systems driven by continuous control inputs where switching is accompanied by a jump in the state. A similar class has been considered in [33], where the authors focus attention on state delayed systems with controlled switches and where useful gradient formulas have been derived, for an application see [34]. See also [35] and [36] for related problems. In contrast, we consider the case where switches are being triggered by the continuous dynamics but the magnitudes of the corresponding state jumps are part of the optimization variables; see also [3] for a gradient-based approach. This family of systems captures phenomena arising in, e.g., cyclically operated batch processes and certain epidemic propagation models.

A simple transfomation relates the optimal control problem for the aforementioned class of systems to another optimal control problems, for which necessary conditions of optimality have been previously derived by the authors [6]. These results make it possible to use conceptual algorithms and their corresponding convergence results, see e.g., [5]. Note that using transformations is a standard approach in optimal control theory and has been used extensively in the past to formulate different results (see e.g., [11], see also [17], where a transformation is used to derive a version of the Maximum principle for a class of hybrid systems).

The outline of the paper is as follows. In Section 2, we formally describe the IHOCP investigated in this contribution. Section 3 contains an equivalent representation of the impulsive hybrid system under consideration and includes an auxiliary optimal control problem for the given IHOCP. In Section 4, we propose a new variant of the hybrid MP for impulsive hybrid systems, namely the Impulsive Hybrid MP. This principle is derived from the MP for hybrid systems with autonomous location transitions and is closely related to the version of the MP proposed in, e.g., [12,13] and to the gradient-based approach to hybrid optimal control problems proposed in [3-5]. In Section 5, we discuss some computational issues of the proposed necessary optimality conditions for IHOCPs. Section 6 summarizes the paper.

\section{Modeling framework and problem formulation}

Let us formally introduce the class of hybrid systems investigated in this paper:

Definition 1. An impulsive hybrid system is a 7-tuple

$$
\mathcal{I H} \mathcal{S}=\{\mathcal{Q}, \mathcal{X}, U, \mathcal{U}, F, \Theta, \mathcal{S}\}
$$

where

$-\mathcal{Q}$ is a finite set of locations; 
- $\mathcal{X}=\left\{\mathcal{X}_{q}\right\}_{q \in \mathcal{Q}}$ is a collection of state sets with $\mathcal{X}_{q} \subseteq \mathbb{R}^{n}$;

$-U \subseteq \mathbb{R}^{m}$ is a control set;

$-\mathcal{U}$ is a set of admissible control functions;

$-F=\left\{f_{q}\right\}_{q \in \mathcal{Q}}$ is a family of vector fields $f_{q}:\left[0, t_{f}\right] \times \mathcal{X}_{q} \times U \rightarrow \mathbb{R}^{n}$;

- $\Theta=\left\{\Theta_{q}\right\}_{q \in \mathcal{Q}}$ is a collection of maximal constant amplitudes (state jumps);

$-\mathcal{S}$ is a subset of $\Xi$, where

$$
\Xi:=\left\{\left(q, x, q^{\prime}, x^{\prime}\right), q, q^{\prime} \in \mathcal{Q}, x \in \mathcal{X}_{q}, x^{\prime} \in \mathcal{X}_{q^{\prime}}\right\} .
$$

In the following, we consider only impulsive hybrid systems $\mathcal{I H S}$ that satisfy the following assumptions:

A1 The functions $f_{q}(t, \cdot, \cdot)$, where $q \in \mathcal{Q}$, are continuously differentiable

A2 There exists a constant $K<\infty$ such that $\left\|\partial f_{q}(t, x, u) / \partial x\right\|<K$ for all $(t, x, u) \in\left[0, t_{f}\right] \times \mathcal{X}_{q} \times U$ for all $q \in \mathcal{Q}$

A3 The control set $U$ is compact and convex

Moreover, we assume that smooth functions $m_{q, q^{\prime}}: \mathbb{R}^{n} \rightarrow \mathbb{R}, q, q^{\prime} \in \mathcal{Q}$ with nonzero gradients are given such that the hypersurfaces

$$
M_{q, q^{\prime}}:=\left\{x \in \mathbb{R}^{n}: m_{q, q^{\prime}}(x)=0\right\}
$$

are pairwise disjoint. Note that in this case a hypersurface $M_{q, q^{\prime}}$ characterizes the set $\mathcal{S}$ at which a switch from location $q$ to location $q^{\prime}$ can take place. Evidently, $M_{q, q^{\prime}}$ is the projection of $\mathcal{S}$ on the product space $\mathcal{X}_{q} \times \mathcal{X}_{q^{\prime}}$. The set of admissible control functions from Definition 1 is taken as

$$
\mathcal{U}:=\left\{u(\cdot) \in \mathbb{L}_{\infty}^{m}\left(0, t_{f}\right): u(t) \in U_{q}, \text { a.e. on }\left[0, t_{f}\right]\right\} .
$$

By $\mathbb{L}_{\infty}^{m}\left(0, t_{f}\right)$ we denote the standard Lebesque space of measurable and essentially bounded functions. Note that the pair $(q, x(t))$ represents the hybrid state at time $t$, where $q$ is a location $q \in \mathcal{Q}$ and $x(t) \in \mathbb{R}^{n}$. Let us introduce some standard spaces, namely, the space $\mathbb{C}_{0}^{\infty}\left(0, t_{f}\right)$ of all $\mathbb{C}^{\infty}$ functions that vanish outside a compact subset of $\left(0, t_{f}\right)$ and the space $\mathcal{D}^{\prime}\left(0, t_{f}\right)$ of generalized functions (Schwartz distributions). Recall that $\mathcal{D}^{\prime}\left(0, t_{f}\right)$ can be considered as a space of linear, sequentially continuous functionals with respect to the convergence on the space $\mathbb{C}_{0}^{\infty}\left(0, t_{f}\right)$. In the following, we define the notion of a hybrid trajectory of an impulsive hybrid system (see e.g., [4],[5]).

Definition 2. A hybrid trajectory of $\mathcal{I H S}$ is a triple $\mathbf{X}=\left(x(\cdot),\left\{q_{i}\right\}, \tau\right)$, where $x(\cdot) \in \mathcal{D}^{\prime}\left(0, t_{f}\right)$ is a discontinuous trajectory, $\left\{q_{i}\right\}_{i=1, \ldots, r}$ is a finite sequence of locations and $\tau$ is the corresponding sequence of switching times

$$
0=t_{0}<\cdots<t_{i}<\cdots<t_{r}=t_{f}
$$

such that for each $i=1, \ldots, r$ there exists $u(\cdot) \in \mathcal{U}$ such that:

- $x(0)=x_{0} \notin \bigcup_{q, q^{\prime} \in \mathcal{Q}} M_{q, q^{\prime}}$ and $x_{i}(\cdot)=\left.x(\cdot)\right|_{\left(t_{i-1}, t_{i}\right)}$ is an absolutely continuous function on $\left(t_{i-1}, t_{i}\right)$; 


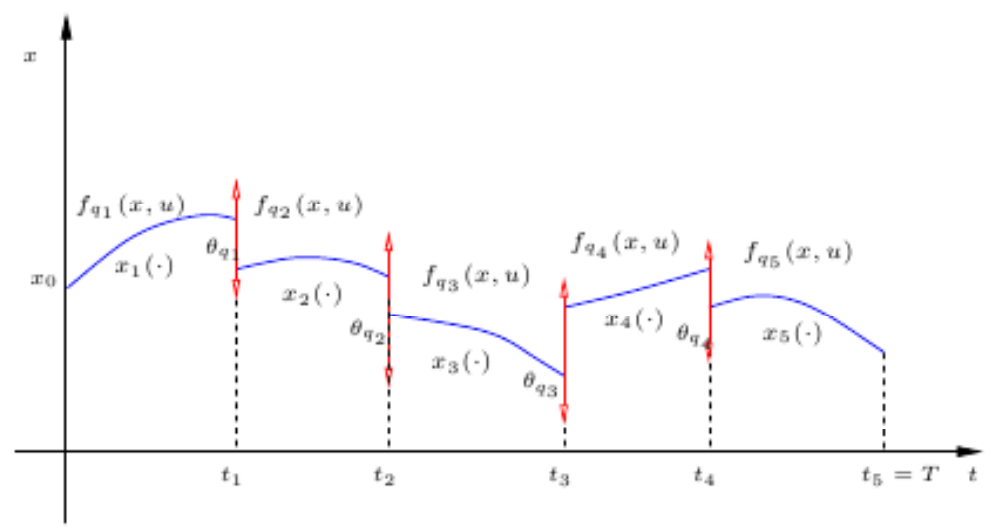

Fig. 1. An example of execution with 4 switches $(r=5)$.

- $x\left(t_{i}\right) \in M_{q_{i}, q_{i+1}}$ for $i=1, \ldots, r-1$;

- $\dot{x}_{i}(t)=f_{q_{i}}\left(t, x_{i}(t), u(t)\right)+\theta_{q_{i}} \delta\left(t-t_{i}\right)$ for almost all $t \in\left[t_{i-1}, t_{i}\right]$, where $\delta$ is the Dirac function and $\left\|\theta_{q_{i}}\right\| \leq \Theta_{q_{i}}$.

The derivative $\dot{x}_{i}(\cdot)$ in Definition 2 is considered as a weak derivative of the generalized function $x_{i}(\cdot)$ defined on the full interval $\left[t_{i-1}, t_{i}\right]$. It is also evident that a function $x(\cdot)$ from Definition 2 consists of absolutely continuous parts defined on the open intervals $\left(t_{i-1}, t_{i}\right)$ and involves jumps of magnitude $\theta_{q_{i}}$ at the switching times $t_{i}$, see Figure 1 for an example of the execution. Note that the evolution equation for the trajectory $x(\cdot)$ of a given impulsive hybrid system $\mathcal{I H S}$ can also be represented as follows

$$
\begin{aligned}
& \dot{x}(t)=\sum_{i=1}^{r} \beta_{\left[t_{i-1}, t_{i}\right)}(t) f_{q_{i}}(t, x(t), u(t))+\sum_{i=1}^{r} \theta_{q_{i}} \delta\left(t-t_{i}\right) \text { a.e. on }\left[0, t_{f}\right] \\
& x(0)=x_{0}
\end{aligned}
$$

where $\beta_{\left[t_{i-1}, t_{i}\right)}(\cdot)$ is the characteristic function of the interval $\left[t_{i-1}, t_{i}\right)$

$$
\beta_{\left[t_{i-1}, t_{i}\right)}(t)= \begin{cases}1 & \text { if } t \in\left[t_{i-1}, t_{i}\right) \\ 0 & \text { otherwise }\end{cases}
$$

for $i=1, \ldots, r$. Note that the initial value problem in Equation (1) is also considered in the sense of weak derivatives on the space $\mathcal{D}^{\prime}\left(0, t_{f}\right)$. Under the assumptions presented above, for each $u(\cdot) \in \mathcal{U}$ and all $\left\|\theta_{q_{i}}\right\| \leq \Theta_{q_{i}}, i=1, \ldots, r$, the initial value problem (1) has a unique solution in $\mathcal{D}^{\prime}\left(0, t_{f}\right)$.

Let $f^{0}: \mathbb{R} \times \mathbb{R}^{n} \times \mathbb{R}^{m} \rightarrow \mathbb{R}$ be a continuously differentiable function. Given an impulsive hybrid system $\mathcal{I H S}$ we now formulate a corresponding optimization 
problem, the following Impulsive Hybrid Optimal Control Problem (IHOCP):

$$
\begin{aligned}
& \operatorname{minimize} \sum_{i=1}^{r} \int_{t_{i-1}}^{t_{i}} f^{0}(t, x(t), u(t)) d t \\
& \text { over all trajectories } \mathbf{X} \text { of } \mathcal{I} \mathcal{H} \mathcal{S} .
\end{aligned}
$$

Throughout the paper we assume that the IHOCP (2) has an optimal solution

$$
\left(u^{\text {opt }}(\cdot), \theta^{\text {opt }}, \mathbf{X}^{\text {opt }}(\cdot)\right) \in \mathcal{C}:=\mathcal{U} \times \mathbb{R}^{n \times r} \times \mathcal{D}^{\prime}\left(0, t_{f}\right) \times \mathcal{Q}^{r} \times\left[0, t_{f}\right]^{r}
$$

where $\theta^{o p t}:=\left(\theta_{q_{1}}^{o p t} \ldots \theta_{q_{r}}^{o p t}\right)$ is a matrix representing the optimal jumps.

\section{Optimization of Impulsive Hybrid Systems}

The optimal control problem (2) is an optimization problem formulated on the space $\mathcal{C}$ which involves the space of generalized functions $\mathcal{D}^{\prime}\left(0, t_{f}\right)$. Our aim is to introduce an auxiliary hybrid optimal control problem governed by a hybrid system with autonomous location transitions without jumps in the continuous state see e.g., $[12,13,5-7]$ for further details. For this, consider the following auxiliary initial value problem

$$
\begin{aligned}
& \dot{y}(t)=\sum_{i=1}^{r} \beta_{\left[t_{i-1}, t_{i}\right)}(t) f_{q_{i}}\left(t, y(t)+\sum_{i=1}^{r} \theta_{q_{i}} \eta\left(t-t_{i}\right), u(t)\right) \text { a.e. on }\left[0, t_{f}\right], \\
& y(0)=x_{0}
\end{aligned}
$$

where $i=1, \ldots, r$ and $\eta(\cdot)$ is the Heaviside step-function. Note that $\eta(\cdot)$ can also be considered as an element of the space $\mathcal{D}^{\prime}\left(0, t_{f}\right)$. Under the assumptions stated in the previous section, the initial value problem (3) has a unique absolutely continuous solution for each $u(\cdot) \in \mathcal{U}$ (see, e.g., $[9,22])$. Next we consider $y(\cdot)$ as an element of the Sobolev space $x(\cdot) \in \mathbb{W}_{n}^{1, \infty}\left(0, t_{f}\right)$, i.e., the space of absolutely continuous functions with essentially bounded derivatives. We are now able to formulate our first equivalence result.

Theorem 1. Under the above-mentioned assumptions $A 1-A 3$, the (unique) solution $x(\cdot) \in \mathcal{D}^{\prime}\left(0, t_{f}\right)$ of the initial value problem (1) can be represented in the following form:

$$
x(t)=y(t)+\sum_{i=1}^{r} \theta_{q_{i}} \eta\left(t-t_{i}\right),
$$

where $y(\cdot) \in \mathbb{W}_{n}^{1, \infty}\left(0, t_{f}\right)$ is a (unique) solution to the initial value problem (3).

Proof. Since the weak derivative of the Heaviside step-function $\eta\left(t-t_{i}\right)$ is equal to the Dirac function $\delta\left(t-t_{i}\right)$, the weak derivative of the right-hand side of (4) is

$$
\dot{y}(t)+\sum_{i=1}^{r} \theta_{q_{i}} \delta\left(t-t_{i}\right) .
$$


For an absolutely continuous function $y(\cdot)$ the weak derivative of $y(\cdot)$ coincides with the classical derivative. Using equation (4), the initial value problem (1) can be written in the following form

$$
\begin{aligned}
& \dot{x}(t)=\dot{y}(t)+\sum_{i=1}^{r} \theta_{q_{i}} \delta\left(t-t_{i}\right)=\sum_{i=1}^{r} \beta_{\left[t_{i-1}, t_{i}\right)}(t) f_{q_{i}}(t, y(t) \\
& \left.+\sum_{i=1}^{r} \theta_{q_{i}} \eta\left(t-t_{i}\right), u(t)\right)+\sum_{i=1}^{r} \theta_{q_{i}} \delta\left(t-t_{i}\right) \\
& =\sum_{i=1}^{r} \beta_{\left[t_{i-1}, t_{i}\right)}(t) f_{q_{i}}(t, x(t), u(t))+\sum_{i=1}^{r} \theta_{q_{i}} \delta\left(t-t_{i}\right)
\end{aligned}
$$

Moreover, for $t=0$ we obtain $x(0)=y(0)$. The uniqueness arguments for solutions of the initial value problems (1) and (3) complete the proof.

It is necessary to stress that the proposed representation (4) can also be considered as a transformation of values. This transformation eliminates state jumps at the switching times $t_{i} \in \tau$ from the original system (1). From the affine structure of (4) we can deduce the following simple characterization of (4) with respect to solutions of the above initial value problems.

Theorem 2. The transformation (4) from Theorem 1 is a bijective mapping $\mathcal{D}^{\prime}\left(0, t_{f}\right) \rightarrow \mathbb{W}_{n}^{1, \infty}\left(0, t_{f}\right)$ and the solutions $x(\cdot) \in \mathcal{D}^{\prime}\left(0, t_{f}\right)$ and $y(\cdot) \in \mathbb{W}_{n}^{1, \infty}\left(0, t_{f}\right)$ of the initial value problems (1) and (3) are related by equation (4).

Our results, namely, Theorem 1 and Theorem 2, give rise to the study of an auxiliary hybrid system with autonomous location transitions. Recall the corresponding definition.

Definition 3. A hybrid system with autonomous location transitions is a 6 tuple

$$
\mathcal{H}=\left\{\mathcal{Q}, \mathcal{X}, U, \mathcal{U}, F, \mathcal{S}^{a}\right\}
$$

where

$-\mathcal{Q}$ is a finite set of locations;

$-\mathcal{X}=\left\{\mathcal{X}_{q}\right\}_{q \in \mathcal{Q}}$ is a collection of state sets with $\mathcal{X}_{q} \subseteq \mathbb{R}^{n}$;

- $U \subseteq \mathbb{R}^{m}$ is a control set;

$-\mathcal{U}$ is a set of admissible control functions;

- $F=\left\{f_{q}\right\}_{q \in \mathcal{Q}}$ is a family of vector fields $f_{q}:\left[0, t_{f}\right] \times \mathcal{X}_{q} \times U \rightarrow \mathbb{R}^{n}$;

- $\mathcal{S}^{a}$ is a subset of $\Xi_{a}$, where

$$
\Xi^{a}:=\left\{\left(q, y, q^{\prime}, y^{\prime}\right), q, q^{\prime} \in \mathcal{Q}, y \in \mathcal{X}_{q}, y^{\prime} \in \mathcal{X}_{q^{\prime}}\right\}
$$

Moreover, a hybrid trajectory of $\mathcal{H}$ is a triple $\mathbf{Y}=\left(y(\cdot),\left\{q_{i}\right\}^{a}, \tau^{a}\right)$, where $y(\cdot):[0, T] \rightarrow \mathbb{R}^{n}$ and for each $i=1, \ldots, r$, there exists $u(\cdot) \in \mathcal{U}$ such that: 
- $y(0)=x_{0}$ and $y_{i}(\cdot)=\left.y(\cdot)\right|_{\left(t_{i-1}, t_{i}\right)}$ is an absolutely continuous function on $\left(t_{i-1}, t_{i}\right)$ continuously prolongable to $\left[t_{i-1}, t_{i}\right], i=1, \ldots, r$;

- $\dot{y}_{i}(t)=f_{q_{i}}\left(t, y_{i}(t), u(t)\right)$ for almost all $t \in\left[t_{i-1}, t_{i}\right], i=1, \ldots, r$;

- the following switching condition $\left(y_{i}\left(t_{i}\right), y_{i+1}\left(t_{i}\right)\right) \in S_{q_{i}, q_{i+1}}^{a}$ holds for each value $i=1, \ldots, r-1$, where

$$
S_{q, q^{\prime}}^{a}:=\left\{\left(y, y^{\prime}\right) \in \mathcal{X}_{q} \times \mathcal{X}_{q^{\prime}}:\left(q, y, q^{\prime} y^{\prime}\right) \in \mathcal{S}^{a}\right\}
$$

is a switching set from location $q \in \mathcal{Q}$ to location $q^{\prime} \in \mathcal{Q}$ with $y=y^{\prime}$ meaning the absence of jumps in the continuous state.

Under assumptions of Section 2, the switching sets $S_{q_{i}, q_{i+1}}^{a}$ can be characterized by the following constructive conditions

$$
m_{q_{i}, q_{i+1}}\left(y(t)+\sum_{i=1}^{r} \theta_{q_{i}} \eta\left(t-t_{i}\right)\right)=0, i=1, \ldots, r-1,
$$

where functions $m_{q_{i}, q_{i+1}}$ defines the manifolds $M_{q_{i}, q_{i+1}}$ from Definition 2 .

For the system described by the initial value problem (3), we now formulate the following optimal control problem (see also [5-7])

$$
\operatorname{minimize} \sum_{i=1}^{r} \int_{t_{i-1}}^{t_{i}} f^{0}\left(t, y(t)+\sum_{i=1}^{r} \theta_{q_{i}} \eta\left(t-t_{i}\right), u(t)\right) d t
$$

over all trajectories $\mathbf{Y}$ of $\mathcal{H}$.

We assume that the optimal control problem (5) has a solution

$$
\left(u^{\text {opt }}(\cdot), \theta^{\text {opt }}, \mathbf{Y}^{\text {opt }}(\cdot)\right) \in \mathcal{U} \times \mathbb{R}^{n \times r} \times \mathbb{W}_{n}^{1, \infty}\left(0, t_{f}\right) \times \mathcal{Q}^{r} \times\left[0, t_{f}\right]^{r} .
$$

The following result establishes the relations between the two optimization problems (2) and (5).

Theorem 3. Suppose that problems (2) and (5) have both optimal solutions. Under the assumptions $A 1-A 3$, every optimal solution $\left(u^{\text {opt }}(\cdot), \theta^{\text {opt }}, \mathbf{Y}^{\text {opt }}\right)$ of problem (5) defines the corresponding optimal solution $\left(u^{\text {opt }}(\cdot), \theta^{\text {opt }}, \mathbf{X}^{\text {opt }}(\cdot)\right)$ for problem (2), where

$$
\begin{aligned}
& \left\{q_{i}\right\}^{a}=\left\{q_{i}\right\}, \tau^{a}=\tau, \\
& x^{o p t}(t)=y^{o p t}(t)+\sum_{i=1}^{r} \theta_{q_{i}}^{o p t} \eta\left(t-t_{i}^{o p t}\right)
\end{aligned}
$$

Here $t_{i}^{\text {opt }}$ is an element of the optimal sequence $\tau^{\text {opt }}$ and $\theta_{q_{i}}^{\text {opt }}$ are optimal jumps in the original IHOCP (2).

Note that Theorem 3 is an immediate consequence of Theorem 2.

As evident from the main Definitions 1 and 3, the class of hybrid control systems with autonomous location transitions is a subclass of impulsive hybrid 
systems. Definition 1 describes hybrid dynamical systems with discontinuous state trajectories. Moreover, the control variable of an impulsive hybrid system $\mathcal{I H S}$ includes external inputs and magnitudes of the jumps in the state. On the other hand, the proposed transformation (4) and the obtained results, namely, Theorems 1-3 make it possible to reduce the general sophisticated IHOCP (2) to the auxiliary optimal control problem of the type (5).

\section{The Impulsive Hybrid Maximum Principle}

For both hybrid systems (1) and (3) we introduce the extended control vector $v(\cdot):=(u(\cdot), \theta)$, where $\theta:=\left(\theta_{q_{1}}, \ldots, \theta_{q_{r}}\right)$. An admissible extended control vector $v(\cdot)$ satisfies the conditions

$$
u(\cdot) \in \mathcal{U},\left\|\theta_{q_{i}}\right\| \leq \Theta_{q_{i}}, i=1, \ldots, r .
$$

An optimal extended control vector is denoted by $v^{o p t}(\cdot)$ and the corresponding elements of this vector are denoted by $u^{o p t}(\cdot)$ and $\theta^{o p t}$. Now we apply the known MP $[6,7]$.

Theorem 4. Let the functions $f^{0}, f_{q_{i}}$ be continuously differentiable and the optimal control problem (5) be regular. Then there exist a function $\psi_{i}(\cdot)$ from $\mathbb{W}_{n}^{1, \infty}\left(0, t_{f}\right)$ and a non-zero vector $a=\left(a_{1} \ldots a_{r-1}\right)^{T} \in \mathbb{R}^{r-1}$ such that

$$
\begin{aligned}
& \dot{\psi}_{i}(t)=-\frac{\partial H_{q_{i}}\left(y_{i}^{o p t}(t), v^{o p t}(t), \psi(t)\right)}{\partial\left(y+\sum_{j=i}^{r} \theta_{q_{j}}\right)} \text { a. e. on }\left(t_{i-1}^{o p t}, t_{i}^{o p t}\right), \\
& \psi_{r}\left(t_{f}\right)=0
\end{aligned}
$$

and

$$
\begin{aligned}
& \psi_{i}\left(t_{i}^{o p t}\right)=\psi_{i+1}\left(t_{i}^{o p t}\right)+\left(a_{i} \frac{\partial m_{q_{i}, q_{i+1}}\left(y^{o p t}\left(t_{i}^{o p t}\right)+\sum_{j=i}^{r} \theta_{q_{j}}^{o p t}\right)}{\partial\left(y+\sum_{j=i}^{r} \theta_{q_{j}}\right)}\right) \\
& i=1, \ldots, r-1
\end{aligned}
$$

Moreover, for every admissible control $v(\cdot)$ the following inequality is satisfied

$$
\begin{aligned}
& \left(\frac{\partial H_{q_{i}}\left(y^{o p t}(t), v^{o p t}(t), \psi(t)\right)}{\partial v},\left(v(t)-v^{o p t}(t)\right)\right) \leq 0 \\
& \text { a.e. on }\left[t_{i-1}^{o p t}, t_{i}^{o p t}\right], i=1, \ldots, r
\end{aligned}
$$

where

$$
H_{q_{i}}(y, v, \psi):=\left(\psi_{i}, f_{q_{i}}\left(t, y+\sum_{i=1}^{r} \theta_{q_{i}} \eta\left(t-t_{i}\right), u\right)\right)-f_{q_{i}}^{0}\left(t, y+\sum_{i=1}^{r} \theta_{q_{i}} \eta\left(t-t_{i}\right), u\right)
$$

is a "partial" Hamiltonian for the location $q_{i} \in \mathcal{Q}, \psi$ is an adjoint vector and $(\cdot, \cdot)$ denotes the corresponding scalar product. 
Using the one-to-one correspondence between the solutions $x^{o p t}(\cdot)$ and $y^{o p t}(\cdot)$ of the initial value problems (1) and (3) established by Theorem 2 and the transformation from Theorem 1, we are now able to formulate the necessary optimality conditions for the original problem (2), namely the IHMP.

Theorem 5. Let functions $f^{0}, f_{q_{i}}$ be continuously differentiable and the optimal control problem (2) be regular. Then there exist a function $p_{i}(\cdot)$ from $\mathbb{W}_{n}^{1, \infty}\left(0, t_{f}\right)$ and a non-zero vector $b=\left(b_{1} \ldots b_{r-1}\right)^{T} \in \mathbb{R}^{r-1}$ such that

$$
\begin{aligned}
& \dot{p}_{i}(t)=-\frac{\partial H_{q_{i}}\left(x_{i}^{o p t}(t), v^{o p t}(t), p(t)\right)}{\partial x} \text { a.e. on }\left(t_{i-1}^{o p t}, t_{i}^{o p t}\right), \\
& p_{r}\left(t_{f}\right)=0
\end{aligned}
$$

and

$$
p_{i}\left(t_{i}^{o p t}\right)=p_{i+1}\left(t_{i}^{o p t}\right)+\left(b_{i} \frac{\partial m_{q_{i}, q_{i+1}}\left(x^{o p t}\left(t_{i}^{o p t}\right)\right)}{\partial x}\right), i=1, \ldots, r-1 .
$$

Moreover, for every admissible control $v(\cdot)$ the following inequalities are satisfied

$$
\begin{aligned}
& \left(\frac{\partial H_{q_{i}}\left(x^{o p t}(t), v^{o p t}(t), p(t)\right)}{\partial u},\left(u(t)-u^{o p t}(t)\right)\right) \leq 0 \\
& \left(\frac{\partial H_{q_{i}}\left(x^{o p t}(t), v^{o p t}(t), p(t)\right)}{\partial \theta},\left(\theta-\theta^{o p t}\right)\right) \leq 0 \\
& \text { a.e. on }\left[t_{i-1}^{o p t}, t_{i}^{o p t}\right], i=1, \ldots, r
\end{aligned}
$$

where $H_{q_{i}}(y, v, p):=\left(p_{i}, f_{q_{i}}(t, x, u)+\theta_{q_{i}} \delta\left(t-t_{i}\right)\right)-f_{q_{i}}^{0}(t, x, u)$ is a "partial" Hamiltonian for the location $q_{i} \in \mathcal{Q}, p$ is an adjoint vector and $(\cdot, \cdot)$ denotes the corresponding scalar product.

Note that Theorem 5 is an immediate consequence of Theorem 4 and the abovementioned ono-to-one correspondence between the solutions of the two initial value problems under consideration. Using the equivalence results from Section 3 , we obtain the necessary optimality conditions for the general IHOCP (2) as a consequence of the MP for the auxiliary problem (5). The presented approach allows to avoid the consideration of generalized functions, weak derivatives and some related sophisticated mathematical techniques, which would be necessary for a direct proof of the above IHMP.

When solving constrained optimal control problems based on some necessary conditions for optimality one can obtain singular solutions. There are two possible scenarios for a singularity: the irregularity of the Lagrange multiplier associated with the cost functional $[9,22]$ and the irregularity of the Hamiltonian. In the latter case the Hamiltonian is not an explicit function of the control function during a time interval. Various supplementary conditions (constraint qualifications) have been proposed under which it is possible to assert that the Lagrange Multiplier Rule (and the corresponding Maximum Principle) holds in "normal" form, i.e., that the first Lagrange multiplier is nonequal to zero. In 
this case the corresponding minimization problem is called regular. We refer to $[1,19,23]$ for theoretical details. Note that some regularity conditions for general constrained optimal control problems can be formulated as controllability conditions for the linearized system [23].

Let us now simplify the Hamiltonian minimization condition (11). Using the given formula for $H_{q_{i}}$, we compute

$$
\frac{\partial H_{q_{i}}\left(x^{o p t}(t), v^{o p t}(t), p(t)\right)}{\partial \theta}=p_{i}(t) \delta\left(t-t_{i}^{o p t}\right)
$$

where $t \in\left[t_{i-1}^{o p t}, t_{i}^{o p t}\right]$. Then, the second inequality from Theorem 5 can be writen in the following form

$$
\left(p_{i}(t), \theta_{q_{i}}-\theta_{q_{i}}^{o p t}\right) \delta\left(t-t_{i}^{o p t}\right) \leq 0, t \in\left[t_{i-1}^{o p t}, t_{i}^{o p t}\right]
$$

Integrating (12) over $\left[t_{i-1}^{o p t}, t_{i}^{o p t}\right]$, we obtain

$$
\left(p_{i}\left(t_{i}^{o p t}\right), \theta_{q_{i}}-\theta_{q_{i}}^{o p t}\right) \leq 0 .
$$

Evidently, in the case $p_{i}\left(t_{i}^{\text {opt }}\right)=0$ for any index $i=1, \ldots, r$, the optimal vector of state jumps $\theta^{o p t}$ cannot be found directly by globally minimizing $H_{q_{i}}$. Note that the partial Hamiltonian $H_{q_{i}}$ is an affine function of $\theta_{q_{i}}$. From this it is inferred that in the case of an IHOCP we can have a new kind of singularity, namely, the irregularity of the Hamiltonian with respect to the state jumps. On the other hand, the presented inequality (13) is a condition for a "bang-bang" control with respect to the second part of the extended control vector $v$.

\section{$5 \quad$ Numerical Aspects}

In the previous section we derived a necessary optimality condition (Theorem 4 and Theorem 5) and formulated the Hamiltonian minimization condition in the form of variational inequalities (8) and (11). It is well known that variational inequalities play an important role in optimization theory. We refer to [8] for details. For the numerical treatment of variational inequality see also [24]. It is also well known that the variational inequality (11) is equivalent to the following equation

$$
v^{o p t}(t)=\Pi_{W}\left(v^{o p t}(t)-\alpha \frac{\partial H_{q_{i}}\left(x^{o p t}(t), v^{o p t}(t), p(t)\right)}{\partial v} v^{o p t}(t)\right),
$$

where $\alpha>0$ and $\Pi_{W}$ is a projection operator on the set $U \times U_{\theta}$. Here $U_{\theta}$ is the set of admissible jumps defined by the inequalities $\left\|\theta_{q_{i}}\right\| \leq \Theta_{q_{i}}, i=1, \ldots, r$. To solve (14) one can use a variety of gradient-type algorithms with a projection procedure. Let $N$ be a sufficiently large positive integer number and

$$
G_{N}:=\left\{t^{0}=0, t^{1}, \ldots, t^{N}=T\right\}
$$


be a (possibly nonequidistant) partition of $[0, T]$ with

$$
\max _{0 \leq k \leq N-1}\left|t^{k+1}-t^{k}\right| \leq \epsilon
$$

for a given accuracy constant $\epsilon$. For every control function $u(\cdot) \in \mathcal{U}$ we introduce the piecewise constant control signals $u^{N}(\cdot)$ such that

$$
\begin{aligned}
& u^{n}(t):=\sum_{k=0}^{N-1} \eta_{k}(t) u^{k}, u^{k}=u\left(t^{k}\right), k=0, \ldots, N-1, t \in\left[0, t_{f}\right] \\
& \eta_{k}(t)= \begin{cases}1, & \text { if } t \in\left[t^{k}, t^{k+1}\right] \\
0, & \text { otherwise }\end{cases}
\end{aligned}
$$

Then for an approximate solution of the equation (14) we can consider the following finite-dimensional gradient method

$$
\begin{aligned}
& u^{N, 0} \in U, \theta^{N, 0} \in U_{\theta}, \\
& u^{N,(s+1)}=\Pi_{U}^{1}\left(u^{N, s}-\alpha_{1} \frac{\partial H_{q_{i}}\left(x^{N, s}(t),\left(u^{N, s}(t), \theta^{N, s}\right), p^{N, s}(t)\right)}{\partial u} u^{N, s}\right), \\
& \theta^{N, s+1}=\Pi_{U_{\theta}}^{2}\left(\theta^{N, s}-\alpha_{2} \frac{\partial H_{q_{i}}\left(x^{N, s}(t),\left(u^{N, s}(t), \theta^{N, s}\right), p^{N, s}(t)\right)}{\partial \theta} \theta^{N, s}\right),
\end{aligned}
$$

where $\alpha_{1}, \alpha_{2}$ are some positive constants, $s=0, \ldots, x^{N, s}(\cdot)$ and $p^{N, s}(\cdot)$ are solutions of the corresponding initial and boundary value problems (1) and (9)-(11) in the actual location $q_{i} \in \mathcal{Q}$. Here $\Pi^{1}$ and $\Pi^{2}$ are partial projection operators on the set $U$ and $U_{\theta}$ respectively. Moreover, the iteration of the extended control vector is denoted as $\left(u^{N, s}(\cdot), \theta^{N, s}\right)$. Evidently, $U$ and $U_{\theta}$ are convex sets. Note that in every step of the gradient algorithm (15) we need to solve the corresponding boundary-value problem from Theorem 5. Using inequalities (12) and (13), we can rewrite the second inequality in (15) in the following (integrated) form

$$
\theta^{N, s+1} t_{f}=\Pi_{U_{\theta}}^{2}\left(\theta^{N, s} t_{f}-\alpha_{2} p_{i}^{N, s}\left(t_{i}^{o p t}\right) \theta^{N, s}\right) .
$$

Clearly, the presented inequality (16) must be combined with an effective procedure for estimating the optimal switching time $t_{i}^{\text {opt }}$ for all $i=1, \ldots, r$. One can use the iterative algorithm described in [5] for this purpose.

We refer to [29] for convergence properties of the general gradient-type algorithms. Clearly, instead of piecewise constant control signals one can also use possible approximations of higher order (piecewise linear and so on). Finally, note that the gradient technique (15) is analogous to the gradient-based computational approach proposed in [3-7] for optimization of hybrid systems with autonomous transitions.

\section{Concluding Remarks}

The Hamilton minimization conditions from Theorem 4 and Theorem 5 are presented in the form of variational inequalities. This form is closely related to the 
Weierstraß conditions for a strong minimum (see, e.g., [22]) and to the gradientbased computational approach studied in [3-7]. Finally note that the inequalities conditions (11) from Theorem 5 make it possible to take into consideration some effective methods for numerical treatment of variational inequalities.

Acknowledgements: The authors thank anonymous referees for valuable remarks and suggestions from which the final version of the paper greatly benefited.

\section{References}

1. A.V. Arutyunov and S.M. Aseev, Investigation of the degeneracy phenomenon in the maximum principle for optimal control with state constraints, SIAM Journal on Control and Optimization, 35 (1997), pp. 930 - 952.

2. S.A. Attia, M. Alamir and C. Canudas de Wit, Suboptimal control of switched nonlinear systems unde location and switching constraints. In Proceedings of the 16th IFAC World Congress, Prague, 2005.

3. S.A. Attia, V. Azhmyakov and J. Raisch, State jump optimization for a class of hybrid autonomous systems. In: Proceedings of the 2007 IEEE Multi-conference on Systems and Control, Singapore, 2007, pp. 1408 - 1413.

4. S.A. Attia, V. Azhmyakov and J. Raisch, On gradient methods for hybrid systems optimization, submitted (2007).

5. V. Azhmyakov and J. Raisch, A gradient-based approach to a class of hybrid optimal control problems, in Proceedings of the 2nd IFAC Conference on Analysis and Design of Hybrid Systems, Alghero, 2006, pp. 89 - 94.

6. V. Azhmyakov, S.A. Attia, D. Gromov and J. Raisch, Necessary optimality conditions for a class of hybrid optimal control problems, Lecture Notes in Computer Science, 4416. pp. 637 - 640, Springer, Berlin, 2007.

7. V. Azhmyakov, Optimal control of hybrid and switched systems. In: Proceedings of the IX Chetaev Conference "Analytical Mechanics, Stability and Control of Motion", Irkutsk, 2007, pp. $308-317$.

8. C. Baiocchi and A. Capello, Variational and Quasivariational Inequalities: Application to Free Boundary Problems. Wiley, New York, 1984.

9. L.D. Berkovitz, Optimal Control Theory. Springer, New York, 1974.

10. M.S. Branicky, V.S. Borkar and S.K. Mitter, A unifed framework for hybrid control: model and optimal control theory, IEEE Transactions on Automatic Control, 43 (1998), pp. $31-45$.

11. A.E. Bryson and Y.C. Ho, Applied Optimal Control, Blaisdell Publishing Company, Waltham, 1969.

12. P. Caines and M.S. Shaikh, Optimality zone algorithms for hybrid systems computation and control: From exponential to linear complexity. In: Proceedings of the 13th Mediterranean Conference on Control and Automation, Limassol, 2005, pp. 1292 1297.

13. P. Caines and M.S. Shaikh, Convergence analysis of Hybrid Maximum Principle (HMP) optimal control algorithms. In: Proceedings of the 17th International Symposium on Mathematical Theory of Networks and Systems, Kyoto, 2006, pp. 2083 2088.

14. C. Cassandras, D.L. Pepyne and Y. Wardi, Optimal control of a class of hybrid systems, IEEE Transactions on Automatic Control, 46 (2001), pp. 398 - 415. 
15. F. Clarke and R. Vinter, Optimal multiprocesses, SIAM Journal on Control and Optimization, 27 (1989), pp. 1072 - 1090.

16. F. H. Clarke and R. B. Vinter, Applications of optimal multiprocesses, SIAM Journal on Control and Optimization, 27 (1989), pp. 1048-1071.

17. A. V. Dmitruk and A. M. Kaganovivh, The Hybrid Maximum Principle is a Consequence of Pontryagin Maximum Principle. In: availabe on line at http://www.optimization-online.org/.

18. M. Egerstedt, Y. Wardi and H. Axelsson, Transition-time optimization for switched-mode dynamical systems, IEEE Transactions on Automatic Control, 51 (2006), pp. $110-115$.

19. M.M.A. Ferreira, F.A.C.C. Fontes and R.B. Vinter, Nondegenerate necessary conditions for nonconvex optimal control problems with state constraints, Journal of Mathematical Analysis and Applications, 233 (1999), pp. 116 - 129.

20. A.F. Filippov, Differential Equations with Discontinuous Right-Hand Sides, Kluwer Academic Publishers, Dordrecht, 1988.

21. M. Garavello and B. Piccoli, Hybrid necessary priniple, SIAM Journal on Control and Optimization, 43 (2005), pp. $1867-1887$.

22. A.D. Ioffe and V.M. Tichomirov, Theory of Extremal Problems, North Holland, Amsterdam, 1979.

23. J. Jahn, Introduction to the Theory of Nonlinear Optimization, Springer, Berlin, 2007.

24. A. Kaplan, and R. Tichatschke, Stable Methods for Ill-Posed Variational Problems, Akademie Verlag, Berlin, 1994.

25. J. Lygeros, Lecture Notes on Hyrid Systems, University of Cambridge, Cambridge, 2003.

26. J. Lygeros, M. Quincampoix and T. Rzezuchowski, Impulse differential inclusions driven by discrete measures, Lecture Notes in Computer Science, 4416. pp. 385 398, Springer, Berlin, 2007.

27. B. Piccoli, Hybrid systems and optimal control. In: Proceedings of the 37th IEEE Conference on Decision and Control, Tampa, 1998, pp. $13-18$.

28. B. Piccoli, Necessary conditions for hybrid optimization. In: Proceedings of the 38th IEEE Conference on Decision and Control, Phoenix, 1999, pp. $410-415$.

29. E. Polak, Optimization, Springer, New York, 1997.

30. A. Rantzer, On relaxed dynamic programming in switching systems. In: IEE Proceedings on Control Theory and Applications, 153 (2006), pp. 567 - 574.

31. M.S. Shaikh and P. E. Caines, On the Hybrid Optimal Control Problem: Theory and Algorithms. In: IEEE Trans. On Aut. Control, 52 (2007), pp. $1587-1603$.

32. H.J. Sussmann, A maximum principle for hybrid optimization. In Proceedings of the 38th IEEE Conference on Decision and Control, Phoenix, 1999, pp. $425-430$.

33. E. Verriest and F. Delmotte and M. Egerstedt, Optimal impulsive control of point delay systems with refractory period. In Proceedings of the 5th IFAC workshop on Time Delay Systems, Leuven, Belgium, 2004,.

34. E. Verriest and F. Delmotte and M. Egerstedt, Control of epidemics by vaccination. In Proceedings of the American Control Conference, Portland, 2005, pp. 985-990.

35. X. Xu and P.J. Antsaklis, Optimal Control of Hybrid autonomous Systems with State Jumps. In: Proceedings of the American Control Conference, Denver, 2003, pp. 5191-5196.

36. X. Xu and P. J. Antsaklis, Results and Perspectives on Computational Methods for Optimal Control of Switched Systems, Lecture Notes in Computer Science, HSCC. pp. 540-555, Springer, Berlin, 2003. 